\title{
MODELOS POLINOMIAIS PARA REPRESENTAR O PERFIL E O VOLUME DO FUSTE DE Eucalyptus urophylla NA REGIÃO NORTE DO ESTADO DE GOIÁS
}

\author{
Eder Pereira Miguel ${ }^{1}$, Sebastião do Amaral Machado², Afonso Figueiredo Filho ${ }^{3}$, Julio Eduardo Arce ${ }^{4}$ \\ ${ }^{1}$ Eng. Florestal, Doutorando do Curso de Eng. Florestal, Unb, Brasília, DF, Brasil - miguelederpereira@ gmail.com \\ ${ }^{2}$ Eng. Florestal, Ph.D., Prof. Sênior do Depto. de Ciências Florestais, UFPR, Curitiba, PR, Brasil - samachado@ufpr.br \\ ${ }^{3}$ Eng. Florestal, Dr., Prof. do Depto. de Eng. Florestal, UNICENTRO, Irati, PR, Brasil - afig@ufpr.br \\ ${ }^{4}$ Eng. Florestal, Dr., Prof. do Depto. de Ciências Florestais, UFPR, Curitiba, PR, Brasil - jarce@ floresta.ufpr.br \\ Recebido para publicação: 01/02/2009 - Aceito para publicação: 26/08/2010
}

\begin{abstract}
Resumo
A possibilidade de estimar o volume madeirável de um determinado empreendimento florestal e paralelamente a isso fazer a predição de múltiplos produtos advindos do mesmo, viabilizam significativos ganhos na administração dos povoamentos florestais. É claro e evidente que a estimativa precisa do volume de um povoamento florestal cada vez mais vem se tornando prática indispensável nas empresas ligadas ao setor madeireiro, e a possibilidade de agregar valores nas florestas, sejam plantadas ou nativas, é possível uma vez que haja meios de diversificar os produtos madeireiros, nos casos em que o resíduo da elaboração de um produto torna-se matéria-prima para elaboração de outro. Esta pesquisa teve como objetivo avaliar a acuracidade dos modelos polinomiais propostos por Schöepfer (1966), Kozak et al. (1969) e Hradetzky (1976), para estimativa de diâmetros e volumes ao longo do fuste de Eucalyptus urophylla, em plantio localizado no município de Niquelândia, no estado do Goiás. A base de dados foi composta por 48 árvores cubadas rigorosamente pelo método de Smalian. A avaliação da precisão dos modelos foi feita através da análise de resíduos, Erro Padrão Residual $\left(\mathrm{S}_{\mathrm{yx}}\right)$ e Coeficiente de Determinação $\left(\mathrm{R}^{2}\right)$. O modelo que gerou as melhores estatísticas para a estimativa dos diâmetros ao longo do fuste foi o modelo proposto por Hradetzky (1976). Já para a estimativa do volume total com e sem casca, o polinômio do $5^{\circ}$ grau proposto por Schöepfer (1966) foi um pouco superior.

Palavras-chave: Modelagem; função de afilamento; volumetria.
\end{abstract}

\begin{abstract}
Polynomial models to represent profile and volume of Eucalyptus urophylla stem in north region of Goias State, Brazil. Timber volume estimates of a certain forest due to its multiple products prediction may increase incomes from forest stands management. It is already clear that precise volume survey of a forest is essential to timber companies in order to increase commercial value to forests (native or planted ones), even by diversification of products, transforming residues into raw material. This research has as its main aim to evaluate accuracy of polynomial models proposed by Schöpfer (1966), Kozak et al. (1969) and Hradetzky (1976) to estimate diameter and volume along Eucalyptus urophylla stem, in the region of Niquelândia, Goiás State. The database was composed by 48 trees that had their absolute volume calculate by the Smalian method. The models precision was evaluated by analysis of residuals, the Standard error of estimate $\left(S_{\mathrm{yx}}\right)$ and the determination coefficient $\left(\mathrm{R}^{2}\right)$. The model that produced the best statistics for diameter estimation along the stem was the model proposed by Hradetzky (1976), as well as the $5^{\text {th }}$ degree model proposed by Schöepfer (1966) proved to be superior to estimate total volume.

Keywords: Modeling; taper function; volumetry.
\end{abstract}

\section{INTRODUÇÃO}

O entendimento da forma do fuste tem sido objeto de muitos estudos, dada a importância desse parâmetro na quantificação do volume. Experiências têm demonstrado que cada espécie, nos seus diversos estádios de desenvolvimento, possui características de afilamento diferenciadas. As árvores, de 
maneira geral, têm em sua base a aparência de um neiloide, passando a se assemelhar a um paraboloide na sua porção mediana, assumindo a forma de cone na sua extremidade superior.

Cada vez mais o mercado exige produtos diversificados e de qualidade. Como os produtos florestais não fogem à regra, faz-se necessário utilizar técnicas de quantificação e qualificação desses produtos, entre as quais se têm os modelos de afilamento, que possibilitam a determinação do volume total e comercial de madeira para qualquer diâmetro ou altura especificados.

Existe certo consenso pelos pesquisadores do meio florestal de que o afilamento do fuste das árvores é o decréscimo natural do diâmetro ao longo do tronco e tem efeito direto no volume das árvores, sendo o seu conhecimento de fundamental importância em inventários florestais para o sortimento dos produtos madeiráveis.

Segundo Scolforo (1997), os modelos chamados de funções de afilamento, ou de forma, ou "taper" são uma maneira de se descrever matematicamente o perfil de um tronco. Para tanto, a seção transversal em qualquer posição no tronco é considerada como circular e seu volume é calculado por integração da função.

De acordo com Ahrens; Holbert (1981), uma função de afilamento é uma descrição matemática do perfil longitudinal de um tronco. Assumindo-se que a seção transversal seja circular em qualquer ponto ao longo do tronco, seu volume pode ser obtido por integração daquela função. Assim, em termos geométricos, o tronco é tratado como um sólido de revolução.

O planejamento e a otimização das atividades florestais dependem da precisão das estimativas volumétricas. As estimativas do volume dos bens madeiráveis que possibilite a predição de múltiplos usos da madeira viabilizam e agregam valores significativos, resultando em uma atividade mais rentável para o empreendedor florestal.

A estimativa precisa do volume de um povoamento florestal torna-se cada vez mais importante, à medida que as empresas florestais integram e diversificam suas atividades, visando à diminuição do resíduo, transformando-o em matéria-prima na elaboração de outro produto e assim sucessivamente.

A existência de um modelo que descreva com precisão o perfil do fuste de árvores, na expectativa de estimar o diâmetro a qualquer altura ao longo do fuste e a altura a qualquer diâmetro especificado, permite quantificar o número de toras com bitola e comprimentos pré-definidos ou os múltiplos produtos da madeira. Por meio da integração das funções de afilamento, obtêm-se expressões que permitem estimar o volume desses múltiplos produtos e, consequentemente, de toda a árvore.

Segundo Scolforo (2006), além da flexibilidade para estimar altura de acordo com certo diâmetro e diâmetro de acordo com certa altura, as funções de afilamento apresentam ainda o princípio da compatibilidade entre o volume total e os volumes comerciais estimados. Dessa maneira, existe a garantia de que a soma dos volumes comerciais seja sempre igual ao volume total.

Figueiredo Filho (2005) verificou, em sua pesquisa, a existência de precisões similares entre os valores estimados por equações de volume e por funções de afilamento. Afirmou, no entanto, que é preferível usar equações de afilamento, tendo em vista que essas possibilitam estimar volumes total e parcial entre quaisquer segmentos do fuste.

Diversos modelos de regressão têm sido propostos e empregados para descrever o perfil do fuste, contemplando as variações de forma, segundo a espécie. O melhor modelo depende dos objetivos desejados para cada caso especifico.

De acordo com Machado et al. (2004), o aproveitamento de uma árvore deve ser feito de maneira que se obtenha o maior retorno financeiro possível. Sendo assim, quando uma mesma árvore tem diversos usos finais, o seu valor agregado é maior, pois alcança maior valor no mercado. O conhecimento dessa proporcionalidade tornou-se importante para fins de planejamento da produção em um dado momento ou durante o ciclo de produção da espécie.

Essas diferentes finalidades de uso que uma mesma árvore pode ter são conhecidas no meio florestal como sortimento, e podem ser obtidas através das funções de afilamento, que são instrumentos biométricos básicos para a determinação do critério de retorno de investimento "Rotação Técnica", a qual conjuga a oportunidade do mercado consumidor com as características das dimensões das toras de uma árvore ou povoamento quantitativa e seletivamente.

Segundo Arce (2000), considerando-se que uma árvore será cortada e dividida em toras, para a aplicação de técnicas matemáticas visando a solução de problemas de corte e empacotamento, é necessário que se defina o comprimento útil da tora e alguma função que descreva a redução diamétrica 
da base para o topo. As denominadas funções de afilamento são ferramentas apropriadas para estimar alturas até qualquer diâmetro mínimo comercial, diâmetros a qualquer altura e volumes por compartimento do fuste ou para o total, ou seja, são funções usadas para se fazer sortimento.

Sendo assim, o objetivo deste trabalho foi testar funções de afilamento para moldar o perfil do tronco de Eucalyptus urophylla plantados no norte do estado de Goiás, para estimar diâmetros em diferentes alturas e volumes parciais e total.

\section{MATERIAL E MÉTODOS}

\section{Caracterização da área de estudo e do plantio estudado}

A área de estudo é de propriedade do Grupo Anglo American plc (CODEMIN), localizada no município de Niquelândia, no estado de Goiás, com uma altitude de aproximadamente $535 \mathrm{~m}$ (Figura 1). De acordo com a classificação da EMPRESA BRASILEIRA DE PESQUISA AGROPECUÁRIA (EMBRAPA) (1999), na região do município de Niquelândia predominam solos do tipo Latossolo Vermelho-Amarelo, não hidromórficos, com horizonte B latossólico, apresentando relação molecular Ki baixa, inferior a 1,9, de coloração variando de vermelho ao amarelo e gamas intermediárias. São normalmente muito profundos ou profundos, com sequência de horizontes A, B e C e com transições entre os sub-horizontes difusas e graduais, acentuadamente a bem drenados. Em sua maior parte, os solos da área são álicos, ou seja, com percentagem de saturação de alumínio superior a 50\%, atingindo até valores próximos a 95\%. Apresentam textura média e relevo plano-ondulado.

O clima, segundo a classificação de Köppen, enquadra-se no tipo AW, característico dos climas úmidos tropicais, com duas estações bem definidas: seca, no inverno, iniciando no mês de maio, estendendo-se até o fim de agosto, e úmida, no verão, que tem início em outubro indo até o mês de março, com transições, nas proximidades dos períodos que estão findando, com uma temperatura média anual de $28{ }^{\circ} \mathrm{C}$.

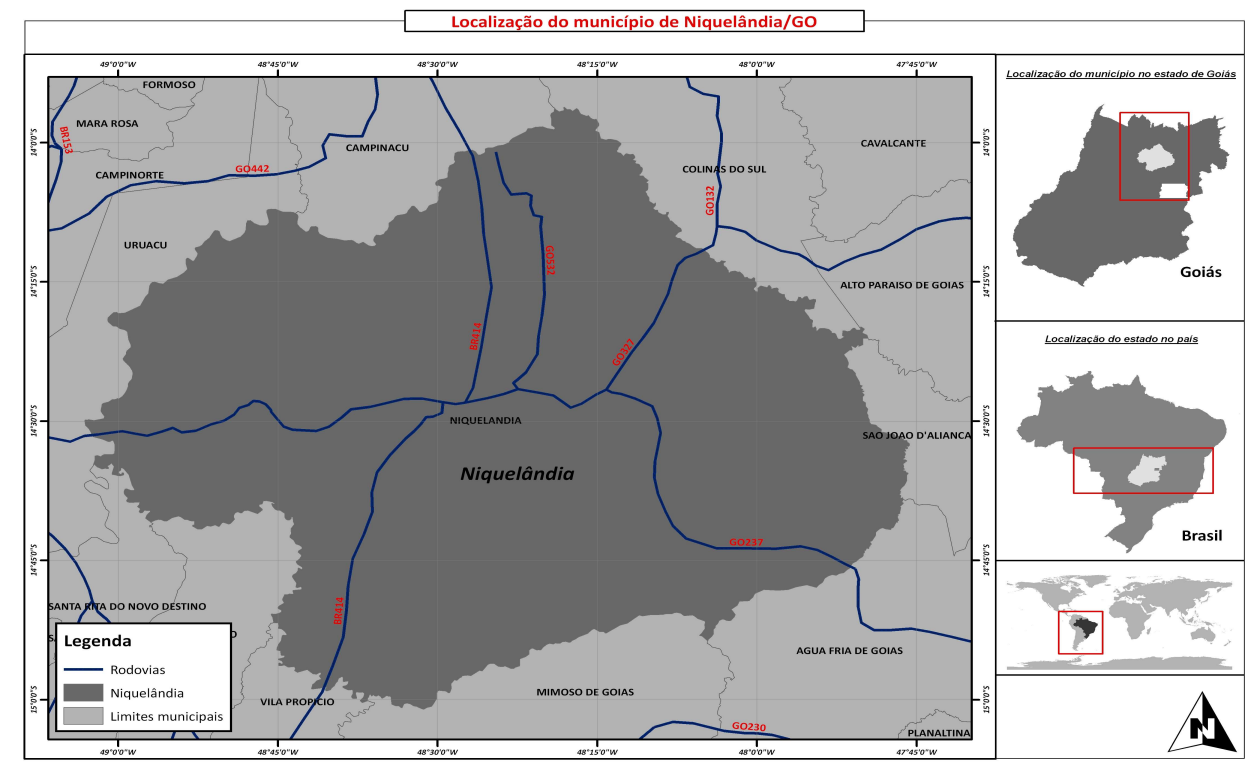

Figura 1. Localização da área de estudo.

Figure 1. Localization of the area of this study.

O plantio onde os dados foram coletados está localizado no município de Niquelândia, região norte do estado de Goiás, constituindo-se de um povoamento clonal de Eucalyptus urophylla, com aproximadamente 5,5 anos de idade. A densidade inicial do povoamento foi de 1666 árvores.ha ${ }^{-1}$ (espaçamento de $3 \mathrm{~m} \times 2 \mathrm{~m}$ ). O povoamento estudado, com base em dados advindos do inventário florestal contínuo de 21 parcelas permanentes de $480 \mathrm{~m}^{2}$, apresenta um DAP médio de 11,3 cm, DAP 
mínimo de $5 \mathrm{~cm}$, DAP máximo de $17,62 \mathrm{~cm}$, altura média de $17,5 \mathrm{~m}$, altura dominante de $20,5 \mathrm{~m}$ e área basal por hectare de $15,66 \mathrm{~m}^{2}$.

A seleção das árvores-amostra para o desenvolvimento das funções de afilamento se baseou na distribuição diamétrica do povoamento, como mostra a tabela 1.

Tabela 1. Frequência de árvores cubadas por classe de diâmetro.

Table 1. Frequency of cubed trees by diameter class.

\begin{tabular}{lc}
\hline Classes de DAP $(\mathbf{c m})$ & $\mathbf{N}^{\circ}$ de árvores cubadas \\
\hline $5-9,9$ & 19 \\
$10-14,9$ & 19 \\
$15-19,9$ & 10 \\
\hline Total & 48 \\
\hline
\end{tabular}

As árvores cubadas foram selecionadas nas proximidades das parcelas permanentes. Para cada árvore amostrada foram realizadas medições da altura total (h), diâmetro à altura do peito (DAP) e altura comercial até o diâmetro mínimo de $3 \mathrm{~cm}$, sendo também medida a espessura da casca em todas as posições de diâmetro medidas ao longo do fuste. Os diâmetros foram medidos nas alturas (m) 0,$1 ; 0,7$; 1,$3 ; 2,0 ; 4,0$ e assim sucessivamente, até atingir a altura comercial estabelecida. Com a tomada de diâmetro nas referidas alturas absolutas, optou-se pelo método de Smalian para cálculo dos volumes, conforme descrito por Husch et al. (1982).

\section{Modelos de afilamentos}

a) Polinômio de quinto grau

O polinômio de quinto grau proposto por Schöepfer (1966) é um dos modelos mais utilizados no Brasil para estimar o volume por sortimento. Segundo Figueiredo Filho et al. (2000), esse modelo tem sido o mais usado para descrever o perfil de Pinus taeda e Pinus elliotti na região Sul do Brasil, sendo expresso por:

$$
\frac{d_{i}}{D A P}=\left[\beta_{0}+\beta_{1}\left(\frac{h_{i}}{h}\right)+\beta_{2}\left(\frac{h_{i}}{h}\right)^{2}+\beta_{3}\left(\frac{h_{i}}{h}\right)^{3}+\beta_{4}\left(\frac{h_{i}}{h}\right)^{4}+\beta_{5}\left(\frac{h_{i}}{h}\right)^{5}\right]
$$

Em que: $\beta_{\text {is }}=$ parâmetros a serem estimados;

$\mathrm{d}_{\mathrm{i}}=$ diâmetros $(\mathrm{cm})$ medidos às alturas $\mathrm{h}_{\mathrm{i}}$ ao longo do fuste;

DAP $=$ diâmetro a $1,3 \mathrm{~m}$ de altura $(\mathrm{cm})$;

$\mathrm{h}=$ altura total $(\mathrm{m})$;

$h_{i}=$ altura nas quais foram medidos os diâmetros $d_{i}$.

O volume da árvore é obtido pela integral do polinômio:

$$
\begin{aligned}
& \mathrm{V}=\mathrm{K} \int_{\mathrm{h}_{1}}^{\mathrm{h}_{2}} \mathrm{~d}_{\mathrm{i}}^{2} \delta h \\
& \quad \mathrm{~V}=\mathrm{KDAP}^{2} \int_{\mathrm{h}_{1}}^{\mathrm{h}_{2}}\left(\mathrm{c}_{0}+\mathrm{c}_{1} \mathrm{~h}_{1}^{\mathrm{p}_{1}}+\mathrm{c}_{2} \mathrm{~h}_{2}^{\mathrm{p}_{2}}+\ldots+\mathrm{c}_{\mathrm{n}} \mathrm{h}_{\mathrm{n}}^{\mathrm{p}_{\mathrm{n}}}\right)^{2} \delta h
\end{aligned}
$$

Em que: $\mathrm{K}=\pi / 40000$;

$\mathrm{p}_{\mathrm{j}}=$ expoentes variando de 1 a 5 ;

$c_{0}=\beta_{0} ; \quad c_{1}=\beta_{1} / \mathrm{h} ; \quad c_{2}=\beta_{2} / \mathrm{h}^{2} ; \ldots ; \mathrm{c}_{5}=\beta_{5} / \mathrm{h}^{5}$.

Resolvendo a integral, tem-se: 


$$
\begin{gathered}
V=K * D A P^{2} *\left[c_{0}^{2} h_{i}+c_{0} c_{1} h_{i}^{2}+\left(\frac{2}{3} c_{0} c_{2}+\frac{1}{3} c_{1}^{2}\right) h_{i}^{3}+\left(\frac{1}{2} c_{0} c_{3}+\frac{1}{2} c_{1} c_{2}\right) h_{i}^{4}+\right] \\
+\left(\frac{2}{5} c_{0} c_{4}+\frac{2}{5} c_{1} c_{3}+\frac{1}{5} c_{2}^{2}\right) h_{i}^{5}+\left(\frac{1}{3} c_{0} c_{5}+\frac{1}{3} c_{1} c_{4}+\frac{1}{3} c_{2} c_{3}\right) h_{i}^{6}+ \\
+\left(\frac{2}{7} c_{1} c_{5}+\frac{2}{7} c_{2} c_{4}+\frac{1}{7} c_{3}^{2}\right) h_{i}^{7}+\left(\frac{1}{4} c_{2} c_{5}+\frac{1}{4} c_{3} c_{4}\right) h_{i}^{8}+ \\
\left.+\left(\frac{2}{9} c_{3} c_{5}+\frac{1}{9} c_{4}^{2}\right) h_{i}^{9}+\frac{1}{5} c_{4} c_{5} h_{i}^{10}+\frac{1}{11} c_{5}^{2} h_{i}^{11}\right]_{h_{1}}^{h_{2}}
\end{gathered}
$$

b) Polinômio de potências fracionárias e inteiras (HRADETZKY, 1976)

Segundo Figueiredo (2005), o modelo de Hradetzky (1976) diferencia-se do modelo proposto por Schöepfer (1996) por apresentar potências fracionárias e inteiras como tentativa de melhor representar o perfil do tronco. Schöepfer (1966) já relatava que o polinômio do $5^{\circ}$ grau, embora represente bem o perfil da árvore, não representa tão bem a porção inferior, já que há mais tortuosidade e sinuosidade na base da árvore que nas partes superiores. Sendo assim, Hradetzky (1976) propôs potências mais apropriadas para a descrição da forma da árvore, apontando como sugestão a construção do modelo pelo método Stepwise, para expoentes variando de 0,005 a 25. O procedimento Stepwise seleciona o conjunto de variáveis independentes que produz a melhor regressão, levando em consideração os valores mais elevados da estatística F.

O procedimento Stepwise inicia-se com um modelo considerando apenas o intercepto; posteriormente, ocorre uma inserção de uma variável independente por vez. A variável independente a ser incluída no modelo é aquela que tem a maior correlação com a variável independente (Y) e maior significância para o teste $\mathrm{F}($ parcial $)$. A inserção de novas variáveis segue o mesmo critério. Esse procedimento possibilita que uma variável independente já adicionada ao modelo possa ser eliminada em um passo seguinte de seleção, por ter se tornado redundante devido à inclusão de outra variável. O número de variáveis retidas é baseado nos níveis de significância, tanto para inclusão quanto para exclusão.

$\mathrm{Na}$ execução desse procedimento, foi considerado o nível de significância pelo teste $\mathrm{F}$, para entrada e saída de variáveis.

Os expoentes utilizados na construção dos modelos por classe de diâmetro através do procedimento Stepwise foram: 0,$005 ; 0,09 ; 0,08 ; 0,07 ; 0,06 ; 0,05 ; 0,04 ; 0,03 ; 0,02 ; 0,01 ; 0,9 ; 0,8 ; 0,7$; 0,$6 ; 0,5 ; 0,4 ; 0,3 ; 0,2 ; 0,1 ; 1 ; 2 ; 3 ; 4 ; 5 ; 10 ; 15 ; 20$ e 25 .

A forma de ajuste do polinômio de potências fracionárias e inteiras é:

$$
\frac{\mathrm{di}}{D A P}=\beta_{0}+\beta_{1}\left(\frac{\mathrm{h}_{\mathrm{i}}}{h}\right)^{\mathrm{p} 1}+\beta_{2}\left(\frac{\mathrm{h}_{\mathrm{i}}}{h}\right)^{\mathrm{p} 2}+\ldots+\beta_{\mathrm{n}}\left(\frac{\mathrm{h}_{\mathrm{i}}}{h}\right)^{\mathrm{pn}}
$$

Em que: $\mathrm{d}_{\mathrm{i}}, \mathrm{DAP}, \mathrm{h}_{\mathrm{i}}, \mathrm{h}, \beta_{\mathrm{i}}$ e $\mathrm{e}_{\mathrm{i}}=\mathrm{já}$ definidos anteriormente;

$\mathrm{p}_{\mathrm{i}}=$ expoentes variando entre 0,005 e 25 .

Isolando $\mathrm{d}_{\mathrm{i}}$, obtém-se a função de afilamento:

$$
d_{i}=D A P\left[\beta_{0}+\beta_{1}\left(\frac{h_{i}}{h}\right)^{p 1}+\beta_{2}\left(\frac{h_{i}}{h}\right)^{p 2}+\ldots+\beta_{n}\left(\frac{h_{i}}{h}\right)^{p n}\right]
$$

Ao simplificar a expressão por: $\mathrm{c}_{0}=\beta_{0}$ e $c_{i}=\left(\frac{\beta_{i}}{h^{p_{j}}}\right)$, em que $\mathrm{i}=1,2, \ldots, \mathrm{n} ;$ e $\mathrm{p}_{\mathrm{j}=\text { expoentes }}$ selecionados através do processo "stepwise", a expressão (5) assume a forma: 


$$
d_{i}=\operatorname{DAP}\left(c_{0}+c_{1} h_{1}^{p_{1}}+c_{2} h_{2}^{p_{2}}+\ldots+c_{n} h_{n}^{p_{n}}\right)
$$

O volume total ou de qualquer porção da árvore (sortimento) é obtido pela resolução da integral do polinômio (6), após sua substituição na expressão (5). O resultado dessa operação é:

$$
\begin{aligned}
& V=K * D A P^{2} *\left[c_{0}^{2} h_{i}+2 c_{0} c_{1}\left(\frac{h_{i}^{\left(p_{1}+1\right)}}{p_{1}+1}\right)+2 c_{0} c_{2}\left(\frac{h_{i}^{\left(p_{2}+1\right)}}{p_{2}+1}\right)+\ldots+2 c_{0} c_{(n-1)}\left(\frac{h_{i}^{\left(p_{(n-1)}+1\right)}}{p_{(n-1)}+1}\right)+\right. \\
& +2 c_{0} c_{n}\left(\frac{h_{i}^{\left(p_{n}+1\right)}}{p_{n}+1}\right)+c_{1}^{2}\left(\frac{h_{i}^{\left(2 p_{2}+1\right)}}{2 p_{1}+1}\right)+2 c_{1} c_{2}\left(\frac{h_{i}^{\left(p_{1}+p_{2}+1\right)}}{p_{1}+p_{2}+1}\right)+\ldots+ \\
& +c_{1} c_{(n-1)}\left(\frac{h_{i}^{\left(p_{1}+p_{(n-1)}+1\right)}}{p_{1}+p_{(n-1)}+1}\right)+2 c_{1} c_{n}\left(\frac{h_{i}^{\left(p_{1}+p_{n}+1\right)}}{p_{1}+p_{n}+1}\right)+c_{2}^{2}\left(\frac{h_{i}^{\left(2 p_{2}+1\right)}}{2 p_{2}+1}\right)+\ldots+ \\
& +2 c_{2} c_{(n-1)}\left(\frac{h_{i}^{\left(p_{2}+p_{(n-1)}+1\right)}}{p_{2}+p_{(n-1)}+1}\right)+2 c_{2} c_{n}\left(\frac{h_{i}^{\left(p_{2}+p_{n}+1\right)}}{p_{2}+p_{n}+1}\right)+\ldots+c_{(n-1)}^{2}\left(\frac{h_{i}^{\left(2 p_{(n-1)}+1\right.}}{2 p_{(n-1)}}\right)+ \\
& \left.\quad+\ldots+2 c_{(n-1)} c_{n}\left(\frac{h_{i}^{\left(p_{(n-1)}+p_{n}+1\right)}}{p_{(n-1)}+p_{n}+1}\right)+c_{n}^{2}\left(\frac{h_{i}^{\left(2 p_{n}+1\right)}}{2 p_{n}+1}\right)\right]_{h_{1}}^{h_{2}}
\end{aligned}
$$

c) Polinômio de Segundo Grau (KOZAK et al., 1969)

Segundo Campos; Leite (2006), este é um dos modelos mais testados na representação do perfil e cálculo do volume do fuste, possivelmente por sua facilidade de ajustamento, além de oferecer certa precisão nas suas estimativas.

A fórmula geral do polinômio de segundo grau de Kozak et al. (1969) é:

$$
\frac{d_{i}^{2}}{D A P^{2}}=b_{0}+b_{1} *\left(\frac{h_{i}}{h}\right)+b_{2} *\left(\frac{h_{i}^{2}}{h^{2}}\right)
$$

Em que: $B_{\mathrm{i}}=$ parâmetros a serem estimados;

$\mathrm{d}_{\mathrm{i}}=$ diâmetros tomados a qualquer altura $\mathrm{h}_{\mathrm{i}}$ ao longo do fuste;

DAP = diâmetro a 1,3 $\mathrm{m}$ de altura;

$\mathrm{h}=$ altura total $(\mathrm{m})$;

$\mathrm{h}_{\mathrm{i}}=$ alturas até qualquer diâmetro $\mathrm{d}_{\mathrm{i}}$ medido ao longo do fuste.

Isolando $\mathrm{d}_{\mathrm{i}}$, obtém-se a função de afilamento:

$d_{i}=D A P \sqrt{\beta_{0}+\beta_{1}\left(\frac{\mathrm{h}_{\mathrm{i}}}{h}\right)+\beta_{2}\left(\frac{\mathrm{h}_{\mathrm{i}}}{h}\right)^{2}}$

O volume total ou de qualquer sortimento da árvore pode ser obtido pela substituição de (8) em (9). Após a resolução da integral, obtém-se como resultado:

$$
V=\frac{\pi}{40000} * D A P^{2} *\left[\left(\beta_{0} *\left(h_{2}-h_{1}\right)\right)+\left(\beta_{1}\left(\frac{h_{2}^{2}-h_{1}^{2}}{2 * h}\right)\right)+\left(\beta_{2} *\left(\frac{h_{2}^{3}-h_{1}^{3}}{3 * h^{2}}\right)\right)\right]
$$

\section{Escolha do melhor modelo ajustado}

A seleção do melhor modelo foi analisada pelas seguintes estatísticas com suas respectivas ordens de importância: 
a) Erro padrão da estimativa (Syx) na escala da variável dependente e em percentagem (Syx\%), sendo que quanto mais próximo de zero melhor, pois ele indica o quão próximo os valores estimados estão dos valores observados.

b) Coeficiente de determinação ajustado $\left(\mathrm{R}^{2} \mathrm{aj}\right)$ : quanto mais próximo de 1 melhor será, pois isso mostra o quanto as variáveis dependentes estão sendo explicadas pelas independentes.

c) Análise de resíduos: os gráficos para as análises de resíduos foram feitos utilizando-se o programa Microsoft Office Excel 2003.

$O R^{2}$ aj foi obtido pela seguinte fórmula:

$$
R^{2} a j .=1-\left[\left(\frac{\text { SQres. }}{\text { SQtotal }}\right) *\left(\frac{n-1}{n-p}\right)\right]
$$

Em que: SQres.= soma de quadrados do resíduo;

SQtotal = soma de quadrados total;

$\mathrm{n}$ = número de observações;

$\mathrm{p}=$ número de coeficientes do modelo matemático.

O erro padrão da estimativa (Syx) foi obtido através da seguinte fórmula:

Em que: Syx = erro padrão da estimativa $(m)$;

$$
\text { syx }=\sqrt{\frac{(Y i-Y e)^{2}}{n-p}} \quad \text { syx } \%=\frac{\text { syx }}{\bar{Y}} * 100
$$

Syx $\%$ = erro padrão da estimativa em porcentagem;

$\mathrm{Yi}=\mathrm{d}_{\mathrm{i}}$ observado ou $v_{\mathrm{i}}$ observado;

$\mathrm{Ye}=\mathrm{d}_{\mathrm{i}}$ estimado ou $v_{\mathrm{i}}$ estimado;

$\overline{\mathrm{Y}}=\mathrm{d}_{\mathrm{i}}$ médio observado ou $v_{\mathrm{i}}$ observado;

$\mathrm{n}=$ número de observações;

$\mathrm{p}=$ número de coeficientes do modelo matemático.

\section{RESULTADOS E DISCUSSÃO}

\section{Ajuste dos modelos de afilamento para expressar o perfil com casca}

Na tabela 2 estão os resultados dos coeficientes dos modelos de afilamento ajustados para a estimativa do diâmetro com casca medido a qualquer altura $h_{i}$ especificada, juntamente com suas estatísticas de ajuste e precisão.

Tabela 2. Coeficientes dos modelos ajustados com suas estatísticas de ajuste e precisão para estimativa do $\mathrm{d}_{\mathrm{i}}$ com casca.

Table 2. Coefficients and statistics of the fitted models for estimating of $\mathrm{d}_{\mathrm{i}}$ outside bark at any height.

\begin{tabular}{lcccc}
\hline Modelos & Coeficientes & Syx $(\mathbf{c m})$ & Syx $(\%)$ & $\mathbf{R}^{2}$ ajustado \\
\hline Kozak et al. & $\mathrm{b}_{0}=1,23907$ & & & \\
& $\mathrm{~b}_{1}=-2,33503$ & 1,070 & 8,604 & 0,930 \\
$\mathrm{~b}_{2}=1,13499$ & & & \\
$\mathrm{~b}_{0}=1,18505$ & & & \\
$\mathrm{~b}_{1}=-3,34565$ & & & \\
$\mathrm{~b}_{2}=13,09940$ & & & \\
Polinômio do $5^{\circ}$ grau & $\mathrm{b}_{3}=-28,93690$ & 0,535 & 4,530 & \\
$\mathrm{~b}_{4}=28,78840$ & & & \\
$\mathrm{~b}_{5}=-10,79120$ & & & \\
$\mathrm{~b}_{0}=1,20174$ & & & 0,983 \\
$\mathrm{~b}_{0,05}=0,46812$ & & & \\
Hradetzky & $\mathrm{b}_{1}=-0,61066$ & 0,523 & 4,420 & \\
& $\mathrm{~b}_{5}=-0,28826$ & & & \\
$\mathrm{~b}_{0,08}=-0,7698$ & & &
\end{tabular}


Analisando a tabela 2, percebe-se que os modelos de Hradetzky e polinômio do $5^{\circ}$ grau apresentaram estatísticas de ajustes e precisão muito semelhantes, para a estimativa dos diâmetros com casca nas diferentes alturas preestabelecidas, com uma ligeira superioridade do modelo de potência fracionária de Hradetzky. Já o modelo de Kozak et al apresentou as piores estatísticas, porém mesmo assim estatísticas a se considerar muita boas.

A figura 2, mostra a distribuição gráfica dos resíduos em percentual ao longo do fuste das árvores.


Figura 2. Distribuição dos resíduos percentuais em função do DAP e da variável estimada para as três equações de afilamento testadas.

Figure 2. Graphic residuals distribution (\%) related to diameters outside bark and volume at different heights along the tree stems.

Analisando os gráficos de dispersão de resíduos, existe certa semelhança entre o polinômio do $5^{\circ}$ grau e o modelo de Hradetzky. No entanto, ambos os modelos apresentam uma ligeira tendência de superestimar nos maiores diâmetros, resultado este que não compromete as suas respectivas estimativas. O modelo de Kozak et al., apesar de não deixar evidenciada a tendência existente nos dois modelos citados anteriormente, apresentou resíduos mais distantes da linha de regressão, sendo assim considerado inferior aos demais.

Devido a essa grande semelhança entre o modelo de Hradetzky e o polinômio do $5^{\circ}$ grau, analisando apenas o gráfico de resíduos visualmente não é possível identificar qual é superior. $\mathrm{O}$ erro padrão da estimativa passou a ser a estatística decisiva para a escolha do modelo que melhor represente as estimativas dos diferentes diâmetros do fuste a diferentes alturas preestabelecidas. Assim, o modelo de Hradetzky foi ligeiramente superior ao polinômio de $5^{\circ}$ grau, e por isso foi o modelo escolhido. 


\section{Ajuste dos modelos de afilamento para a estimativa do $d_{i}$ sem casca}

$\mathrm{Na}$ tabela 3 estão os resultados dos coeficientes dos modelos de afilamento ajustados para a estimativa do diâmetro sem casca ao longo do tronco da árvore, com suas respectivas estatísticas de ajuste e precisão.

Tabela 3. Coeficientes das equações ajustadas com suas estatísticas de ajuste e precisão para a estimativa do $d_{i}$ sem casca.

Table 3. Coefficients and statistics of the taper function equations for estimating of the $\mathrm{d}_{\mathrm{i}}$ inside bark.

\begin{tabular}{lcccc}
\hline Modelos & Coeficientes & Syx $(\mathbf{c m})$ & $\mathbf{S y x}(\boldsymbol{\%})$ & $\mathbf{R}^{2}$ ajustado \\
\hline Kozak & $\mathrm{b}_{0}=1,25600$ & & & \\
$\mathrm{~b}_{1}=-2,41060$ & 0,99 & 8,4 & 0,9390 \\
$\mathrm{~b}_{2}=1,19863$ & & & \\
$\mathrm{~b}_{0}=1,19070$ & & & \\
$\mathrm{~b}_{1}=-3,33394$ & & & \\
$\mathrm{~b}_{2}=12,7750$ & & & \\
$\mathrm{~b}_{3}=-27,61740$ & 0,514 & 4,35 & \\
$\mathrm{~b}_{4}=27,09150$ & & & \\
$\mathrm{~b}_{5}=-10,03340$ & & & \\
Polinômio do 5 $5^{\circ}$ grau & $\mathrm{b}_{0}=1,20628$ & & & 0,9840 \\
$\mathrm{~b}_{0,01}=-12,0439$ & & & \\
$\mathrm{~b}_{0,005}=11,71610$ & 0,500 & 4,29 & \\
$\mathrm{~b}_{1}=-0,56105$ & & & \\
$\mathrm{~b}_{4}=-0,315006$ & & &
\end{tabular}

Analisando a tabela 3, nota-se claramente que os modelos de Hradetzky e polinômio do $5^{\circ}$ grau apresentam estatísticas de ajustes e precisão muito semelhantes para a estimativa do diâmetro sem casca ao longo do fuste, nas diferentes alturas preestabelecidas, como já tinha acontecido para a estimativa desses mesmos diâmetros com casca. Pode-se perceber uma ligeira superioridade do modelo de potência fracionária de Hradetzky. O modelo de Kozak et al., por sua vez, apresentou as piores estatísticas novamente, porém mesmo assim estatísticas a se considerar, dentro de um erro admissível no meio florestal.

A figura 3 mostra a distribuição gráfica dos resíduos para a estimativa dos diâmetros sem casca $\left(\mathrm{d}_{\mathrm{i}}\right)$ nas diferentes alturas preestabelecidas ao longo do fuste da árvore.

Analisando os gráficos de dispersão de resíduos, é visível a mesma semelhança entre o modelo do polinômio do $5^{\circ}$ grau e o modelo de Hradetzky, já vistas na estimativa do $\mathrm{d}_{\mathrm{i}}$ com casca, assim como foi nas estatísticas de ajuste e precisão, novamente apresentando uma ligeira superestimativa nos maiores diâmetros, resultado este que não compromete as suas respectivas estimativas, como mencionado anteriormente. O modelo de Kozak et al. manteve o mesmo padrão de dispersão de resíduos ao longo da linha de regressão, apresentando erros mais distantes dessa mesma linha, sendo assim considerado inferior aos demais.

O erro padrão da estimativa voltou a ser a estatística decisiva para a escolha do melhor modelo, a qual apontou mais uma vez o modelo de Hradetzky como sendo ligeiramente superior ao modelo do polinômio do $5^{\circ}$ grau e também superior ao de Kozak. Esses resultados estão em consonância com a literatura, a qual menciona que a quantidade e a diversidade de potências inteiras e fracionárias propostas por Hradetzky são mais flexíveis que a dos demais modelos, fazendo assim com que este modelo consiga representar de forma mais acurada o perfil do fuste das árvores.

\section{Estimativa do volume com e sem casca}

As integrais das funções de afilamento, conforme citado na metodologia, geraram as equações de volume, com as quais foram calculadas as estatísticas, a fim de selecionar o modelo mais adequado para estimar o volume total com e sem casca.

$\mathrm{Na}$ tabela 4 estão os resultados de ajuste e precisão dos modelos, cujas equações foram integradas para a obtenção da variável volume total com casca. 

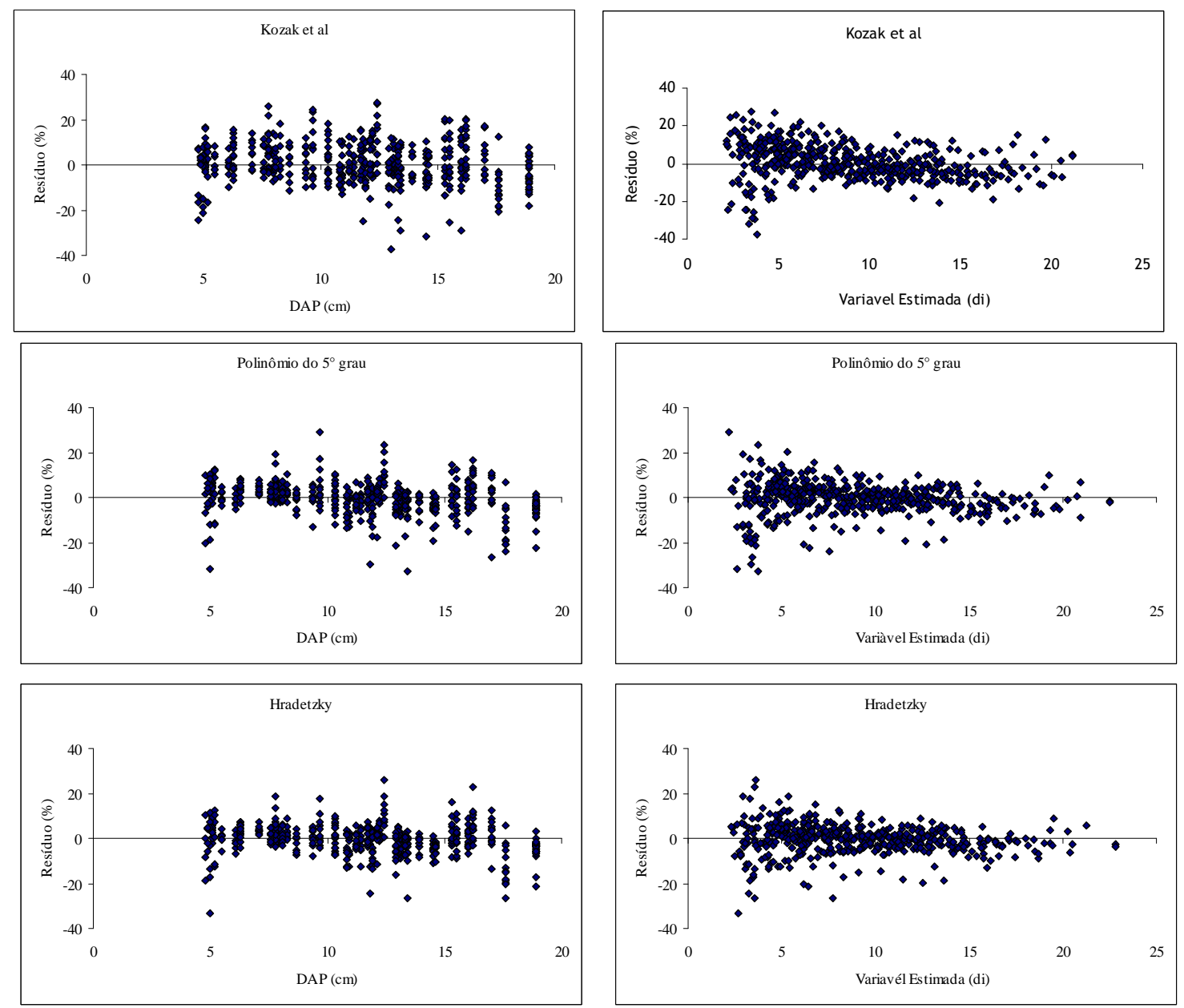

Figura 3. Distribuição dos resíduos percentuais em função do DAP e da variável estimada para as três equações de afilamento testadas.

Figure 3. Residual distribution (\%) over DBH and over the estimated variable for the three tested taper equations.

Analisando a tabela 4, percebe-se que o modelo de Hradetzky e o polinômio do $5^{\circ}$ grau apresentaram estatísticas muito semelhantes e satisfatórias, como já tinha acontecido na estimativa do diâmetro ao longo do fuste, porém agora, na estimativa do volume, o polinômio do $5^{\circ}$ grau apresentou resultados ligeiramente superiores aos de Hradetzky. Já as estatísticas de ajuste e precisão do modelo de Kozak et al. foram as piores dos três modelos ajustados.

Tabela 4. Estatísticas de ajuste e precisão das equações integradas para a obtenção do volume total com casca.

Table 4. Statistics from the integrated taper equations for total volume outside bark estimation.

\begin{tabular}{lccc}
\hline Modelos & $\mathbf{S y x}(\mathbf{m})$ & $\mathbf{S y x}(\boldsymbol{\%})$ & $\mathbf{R}^{\mathbf{2}}$ ajustado \\
\hline Kozak & 0,0101 & 10,13 & 0,930 \\
Polinômio do $5^{\circ}$ grau & 0,00805 & 8,85 & 0,982 \\
Hradetzky & 0,009057 & 9,07 & 0,983 \\
\hline
\end{tabular}

A figura 4 mostra a distribuição gráfica dos resíduos para a estimativa do volume total com casca, sendo um dos critérios para a escolha do melhor modelo de afilamento para a estimativa da variável volume. 

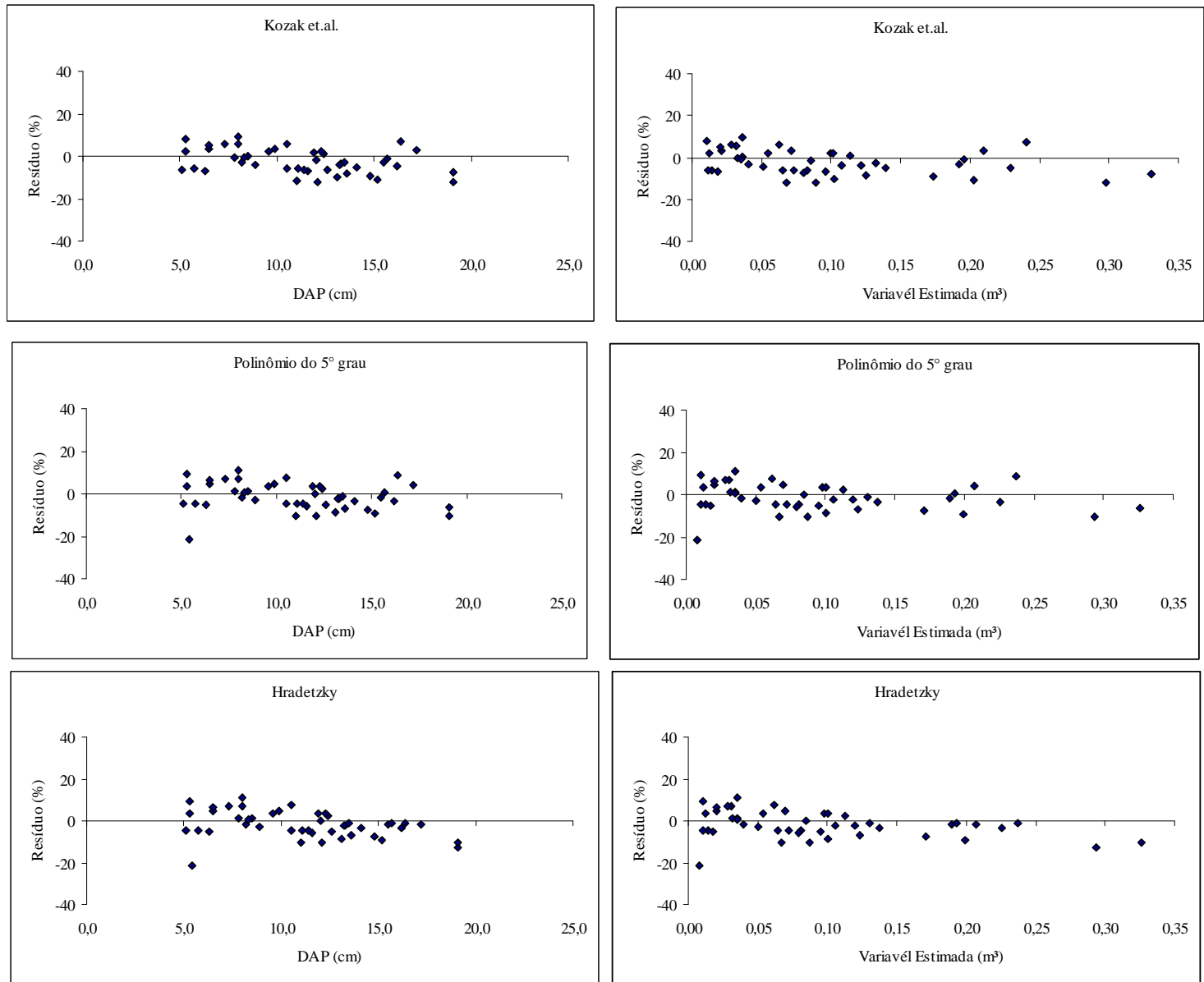

Figura 4. Distribuição dos resíduos percentuais em função do DAP e da variável estimada para as três equações de afilamento testadas.

Figure 4. Residuals distribution over DBH and over the estimated variable for the three tested taper equations.

Analisando os gráficos de resíduos, é perceptível notar que o polinômio do $5^{\circ}$ grau apresentou a melhor distribuição de resíduos ao longo da linha de regressão, não apresentando tendenciosidade. Por outro lado, tanto o modelo de Kozak et al. como o de Hradetzky apresentaram uma ligeira tendência em superestimar os valores nas classes de maiores diâmetros.

Ao analisar o gráfico de resíduos, juntamente com as estatísticas de ajuste e precisão, o modelo de afilamento do polinômio do $5^{\circ}$ grau apresentou-se como o mais apropriado para a estimativa do volume total com casca para este trabalho.

$\mathrm{Na}$ tabela 5 estão os resultados de ajuste e precisão dos mesmos modelos apresentados anteriormente, referentes à integração das equações para a obtenção do volume total sem casca.

Tabela 5. Estatísticas de ajuste e precisão dos modelos de afilamento usados para a obtenção do volume total sem casca.

Table 5. Statistics of the taper equations utilized for estimation of total volume inside bark.

\begin{tabular}{lccc}
\hline Modelos & $\mathbf{S y x}(\mathbf{m})$ & $\mathbf{S y x}(\boldsymbol{\%})$ & $\mathbf{R}^{2}$ ajustado \\
\hline Kozak et al. & 0,01001 & 10,88 & 0,9390 \\
Polinômio do $5^{\circ}$ grau & 0,009385 & 9,45 & 0,9834 \\
Hradetzky & 0,00982 & 9,70 & 0,9840 \\
\hline
\end{tabular}


As estatísticas de ajuste e precisão para a obtenção do volume total sem casca dos referidos modelos manteve a mesma ordem, com uma ligeira superioridade do modelo de afilamento do polinômio do $5^{\circ}$ grau, seguido de perto pelo de Hradetzky. O modelo de Kozak, por sua vez, apresentou as piores estatísticas.

A figura 5 mostra a distribuição gráfica dos resíduos para a estimativa do volume total sem casca.
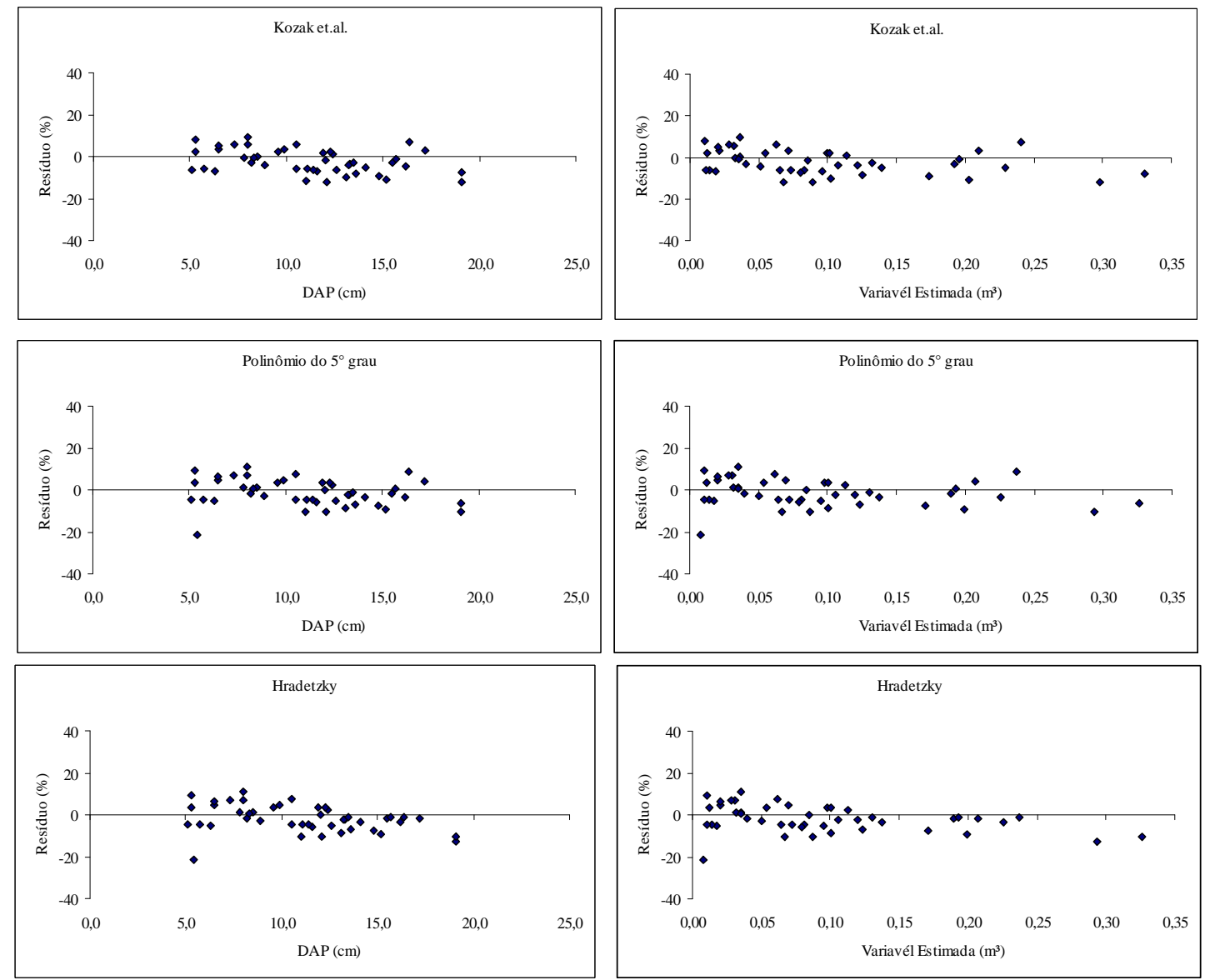

Figura 5. Distribuição dos resíduos percentuais em função do DAP e da variável estimada para as três equações de afilamento testadas.

Figure 5. Distribution of residuals (\%) over DBH and over the estimated variable.

Assim como manteve a ordem das estatísticas de ajuste e precisão para a estimativa do volume total sem casca, nos gráficos de dispersão dos resíduos aconteceu o mesmo, ou seja, o modelo de afilamento do polinômio do $5^{\circ}$ grau mostrou a melhor distribuição de resíduos ao longo da linha de regressão, sem nenhuma tendenciosidade visível, resultado este também encontrado por Schneider et al. (1996), quando testaram 6 modelos de afilamento de tronco para a determinação do volume de um conjunto de árvores de Eucalyptus grandis e chegaram à conclusão de que o modelo polinomial do $5^{\circ}$ grau apresentou os melhores resultados.

O modelo de Hradetzky e o de Kozak et al. já apresentaram uma pequena tendência em superestimar o volume nas árvores de maiores diâmetros, sendo essa tendenciosidade mais marcante no modelo de Kozak. Essa tendenciosidade mais acentuada no modelo de Kozak também foi comprovada por Queiroz (2006), testando modelos de afilamento para povoamentos de bracatinga na Região Metropolitana de Curitiba. Sendo assim, o modelo do Polinômio do $5^{\circ}$ grau foi ligeiramente superior aos demais. 


\section{CONCLUSÕES}

- O modelo de potência fracionária de Hradetzky apresentou as melhores estatísticas para a estimativa de diâmetro ao longo do fuste, tanto com casca como sem casca, seguido de perto pelo modelo polinomial do $5^{\circ}$ grau, ficando as piores estatísticas com o modelo de Kozak et al.

- Para a estimativa do volume total com e sem casca, o modelo polinomial do $5^{\circ}$ grau apresentou as melhores estatísticas de ajuste e precisão, sendo ligeiramente superior ao modelo de potência fracionária de Hradetzky, novamente o modelo de Kozak apresentando os piores ajustes.

- Por esses resultados de ajuste e precisão, o modelo polinomial do $5^{\circ}$ grau foi a equação de afilamento mais apropriada para a estimativa da variável volume total com e sem casca para o plantio de Eucalyptus urophylla localizado na região norte do estado de Goiás.

\section{REFERÊNCIAS}

AHRENS, S.; HOLBERT, D. Uma função para forma de tronco e volume de Pinus taeda L. In: Boletim de pesquisa florestal, unidade regional de pesquisa florestal centro-sul, EMBRAPA, 03, 1981. p. 37 68.

ARCE, J. E. Um sistema de análise, simulação e otimização do sortimento florestal em função da demanda por multiprodutos e dos custos de transporte. 125 p. Tese (Doutorado em Ciência Florestal) - Universidade Federal do Paraná, Curitiba, 2000.

CAMPOS, J. C. C.; LEITE, H. G. Mensuração florestal: perguntas e respostas. 2. ed. Viçosa: Ed. UFV, 2006. $470 \mathrm{p}$.

EMPRESA BRASILEIRA DE PESQUISA AGROPECUÁRIA (EMBRAPA). Centro Nacional de Pesquisa de Solos. Sistema Brasileiro de Classificação de Solos. EMBRAPA, Brasília, 1999.

FIGUEIREDO, E. O. Avaliação de povoamentos de teca (Tectona grandis L.f.) na microrregião do Baixo Rio Acre. 301 f. Dissertação (Mestrado em Engenharia Florestal) - Universidade Federal de Lavras, Lavras, 2005.

FIGUEIREDO FILHO, A.; MACHADO, S. A.; CARNEIRO, M. R. A. Testing accuracy of log volume calculation procedures against water displacement techniques (xylometer). Can. J. For. Res. v. 30, p. 990 - 997, 2000.

HRADETZKY, J. Analyse und interpretation statistisher abranger Keiten. Biometrische Beitrage zu aktuellen forschungs projekten. Baden: Wurttemberg Mitteilungen der FVA, 1976. 146 p. (Abt. Biometric und Informatik, 21).

HUSCH, B.; MILLER, C. I.; BEER, T. W. Forest mensuration. 3. ed. New York: J. Wiley \& Sons, 1982. $402 \mathrm{p}$.

KOZAK, A.; MUNRO, D. D.; SMITH, J. H. G. Taper functions and their aplication in forest inventory. Forest Chronicle, Toronto, v. 45, n. 4, p. 278 - 283, 1969.

MACHADO, S. A.; URBANO, E.; CONCEIÇÃO, M. B.; FIGUEIREDO FILHO, A.; FIGUEIREDO, D. J. Comparação de modelos de afilamento do tronco para diferentes idades e regimes de desbastes em plantações de Pinus oocarpa Schiede. Boletim de Pesquisa Florestal, Colombo, n. 48, p. 41 - 64. jan/jun 2004.

QUEIROZ, D. Avaliação de funções de afilamento e sortimento para Mimosa scabrella Bentham. em povoamentos nativos da Região Metropolitana de Curitiba. 117 f. Dissertação (Mestrado em Engenharia Florestal) - Universidade Federal do Paraná, Curitiba, 2006.

SCHNEIDER, P. R.; FINGER, C. A. G.; KLEIN, J. E. M.; TOTTI, J. A.; BAZZO, J. L. Forma de tronco e sortimento de madeira para Eucalyptus grandis Maiden para o Estado do Rio Grande do Sul. Ciência Florestal, v. 6, n. 1, p. 79 - 88, 1996. 
SCHOEPFER, W. Automatisierung dês Massen, Sorten und Wertberechung stender Waldbestande Schriftenreihe Bad. [S.I.]: Wurtt-Forstl., 1966. Não paginado.

SCOLFORO, J. R. S. Modelagem do crescimento e da produção de florestas plantadas e nativas. Lavras: UFLA/FAEPE, 2006. v. 1. p. 443. 